\title{
MECHANISM OF PRODUCTION OF CANDIDA LESIONS IN RABBITS
}

\author{
V. N. Damodaran and S. C. Chakravarty \\ Department of Pathology and Department of Medical Mycology, \\ Vallabhbhai Patel Chest Institute, University of Delhi, Delhi-7, India
}

\section{Plates X AND XI}

THE respiratory tract is exposed to Candida albicans when this organism colonises the mouth, but serious lung infection rarely occurs. Whether this is due to the low virulence of the organism or to its rapid dispersal from the lungs is not known. Felisati, Bastianini and de Mitri (1959) and Aoyama and Ata (1961) produced lesions in the lungs of rabbits by intratracheal injection of C. albicans, but Kurotchkin and Lim (1933) obtained lung lesions only after sensitising the rabbits previously with injections of killed organisms.

The present experiments were undertaken to clarify the pathogenic effects and mechanism of disposal of $C$. albicans when instilled directly into the lungs in high doses.

\section{MATERIALS AND METHODS}

Male rabbits weighing $1 \frac{1}{2}-2 \mathrm{~kg}$ were used. C. albicans, strain $\mathrm{PCI} / 501 / 9$ isolated from the sputum of a patient and known to be pathogenic for mice, was grown in Sabouraud's glucose broth cultures at $25^{\circ} \mathrm{C}$ for $24 \mathrm{hr}$. The cultures were centrifuged and the organisms re-suspended in normal saline. The suspension consisted only of yeasts with no mycelium, and was adjusted to contain $5-10 \times 10^{7}$ cells per $\mathrm{ml}$ by haemocytometer count.

Methods of inoculation. Rabbits were anaesthetised with ether and $1 \mathrm{ml}$ of a saline suspension of yeasts was injected directly into the trachea exposed through a short skin incision in the midline of the neck. After injection, the animal was held upright for about $5 \mathrm{~min}$. In one group of 29 rabbits the suspension contained $5 \times 10^{7}$ and in the other $10^{8}$ organisms per ml. The animals were killed with ether at intervals after injection of 15, 30 and 45 min., 1, 2, 3, 4, 6, 8, 12, 24, 48, $72 \mathrm{hr}, 4,5,6,7,8,9,10,12$ days and 2, 3, 4, 5, 6, 7, 8,9 wk. They were immediately dissected, and portions of lung, liver, spleen, pancreas, heart and kidney inoculated into Sabouraud's medium. The remaining portions of the organs were fixed in 10 per cent. formalin, and paraffin sections were stained with haematoxylin and eosin, or by the periodic acid-Schiff method, or, where necessary, with haematoxylin and silver methanamine. The morphological forms of $C$. albicans in tissue-yeastlike budding cells, pseudomycelium, and mycelium - were identified according to the criteria of Ajello et al. (1963).

\section{RESULTS}

Mortality. None of the 29 rabbits that received $5 \times 10^{7}$ organisms died. There were three deaths among 29 rabbits that received $10^{8}$ organisms. Of these, one died with extensive bronchopneumonia and bronchial necrosis 48 $\mathrm{hr}$ after injection; one died with severe lesions in the liver and kidney after 
3 days, and one died with small-blood-vessel thrombosis in addition to extensive bronchopneumonia after 5 days.

Fungal culture (tables I and II) of blood was negative throughout in both the groups of animals except in one killed after $30 \mathrm{~min}$.

TABLE I

Fungal culture of blood and viscera in rabbits given $10^{8}$ Candida albicans intratracheally

\begin{tabular}{|c|c|c|c|c|c|c|c|c|c|c|c|c|}
\hline \multirow{2}{*}{$\begin{array}{l}\text { Site of } \\
\text { cultures }\end{array}$} & \multicolumn{12}{|c|}{ Results of culture* after intervals of } \\
\hline & $15 \mathrm{~min}$. & $30 \mathrm{~min}$. & $45 \mathrm{~min}$. & $1 \mathrm{hr}$ & $2 \mathrm{hr}$ & $3 \mathrm{hr}$ & $4 \mathrm{hr}$ & $6 \mathrm{hr}$ & $8 \mathrm{hr}$ & 1-3 days & 4-7 days & $\begin{array}{c}8 \text { days- } \\
9 \mathrm{wk}\end{array}$ \\
\hline Blood & - & + & - & - & - & - & - & - & - & - & - & - \\
\hline Heart & + & + & - & - & - & $\div+$ & + & $+t$ & + & $+t$ & ++ & - \\
\hline Lung & + & + & + & $+t$ & ++ & -+ & + & + & + & ++ & $+t$ & - \\
\hline Kidney & + & + & - & + & + & $\div+$ & + & + & - & +++ & ++ & - \\
\hline Liver & + & ++ & - & ++ & + & + & + & + & + & ++ & ++ & - \\
\hline Spleen & + & + & + & + & + & + & + & + & + & ++ & + & - \\
\hline Pancreas & + & + & + & + & + & + & + & + & + & + & - & - \\
\hline
\end{tabular}

${ }^{*}-=$ Negative culture, $+=$ few colonies, $++=$ heavy growth, $+++=$ very heavy growth.

Within 15 min. of injection, the fungus was found in all the viscera, reaching greatest numbers in the lungs and liver after $1 \mathrm{hr}$ (see fig. 1). By $8 \mathrm{hr}$ the numbers were falling and by 3 days the fungus had disappeared from all the viscera

TABLE II

Fungal cultures of blood and viscera in rabbits given $5 \times 10^{7}$ Candida albicans intratracheally

\begin{tabular}{|c|c|c|c|c|c|c|c|c|c|}
\hline \multirow{2}{*}{$\begin{array}{l}\text { Site of } \\
\text { cultures }\end{array}$} & \multicolumn{9}{|c|}{ Results of culture* after intervals of } \\
\hline & $15 \mathrm{~min}$. & $30 \mathrm{~min}$. & $45 \mathrm{~min}$. & $1 \mathrm{hr}$ & $3 \mathrm{hr}$ & $6 \mathrm{hr}$ & $8 \mathrm{hr}$ & 1-3 days & 4 days -9 wk \\
\hline Blood & - & - & - & - & - & - & - & - & - \\
\hline Heart & + & + & - & - & + & + & + & - & - \\
\hline Lung & + & + & + & ++ & ++ & ++ & ++ & + & - \\
\hline Kidney & + & + & + & + & + & + & + & + & - \\
\hline Liver & - & + & + & ++ & ++ & ++ & - & - & - \\
\hline Spleen & - & + & + & + & + & + & + & - & - \\
\hline Pancreas & - & + & + & + & + & + & + & - & - \\
\hline
\end{tabular}

* $-=$ Negative culture, $+=$ few colonies, $++=$ heavy growth.

except the kidneys and the lungs, where in the animals receiving the higher dose $\left(10^{8}\right.$ cells) it persisted for up to 5 days. Despite this persistence, neither in the lungs nor in other viscera did chronic lesions appear. Micro-abscesses developed in the myocardium, kidney and liver where they appeared earlier and persisted longer in animals given $10^{8}$ yeasts. Bronchopneumonia and necrosis of the bronchial mucosa (fig. 2) occurred only with the higher dose of C. albicans.

Morphology of organisms. The injected yeasts began to transform into 
pseudomycelia within $1 \mathrm{hr}$ after injection, and by $8 \mathrm{hr}$ the majority had grown into long mycelia (fig. 3) with few persisting yeast forms. This change of form was seen in all the organs, but in the lungs yeast forms persisted up to 5 days. Only the mycelial form invaded the alveolar septa, the bronchial wall and small blood vessels (figs. 4 and 5), where mycelial masses blocked capillaries and small blood vessels, resulting in thrombosis and focal areas of necrosis. The yeast forms were easily phagocytosed and destroyed by the alveolar macrophages, but the pseudomycelial forms were practically unaffected. Those that were phagocytosed by macrophages or polymorphonuclear leucocytes grew out of the cell into long mycelial filaments which penetrated neighbouring cells.

Once fungus reached the kidney (fig. 6) it proliferated rapidly as mycelial forms in the glomerular tuft where only a few yeast forms persisted. Mycelia grew along the lumen of the tubules and penetrated through the epithelium into the interstitial tissue and adjacent tubules with resulting tubular necrosis (fig. 6) and abscess formation.

Exudate. Polymorphonuclear leucocytes appeared 1-2 hr after injection but showed little or no phagocytic activity. Later they increased in number and formed suppurative lesions in the lungs, myocardium and kidneys. Few large monocytes (fig. 1) were present initially in the lungs, but they increased with time, actively phagocytosing and subsequently digesting both yeast and mycelial forms with a resulting decrease in the number of organisms in the lesions after 3 days.

In 5-6 days focal granulomata formed in the kidney leading to wedgeshaped scars in the cortex after 10 days.

\section{DisCUSSION}

The rapid disappearance of the fungus from the blood and its persistence in the kidneys may be due to its continuous clearance from the blood by the kidney. Recovery of fungus from various organs, blood-vessel invasion in the lungs, multiple abscesses in the myocardium, liver and kidney, and the emergence of the organisms from the glomerular tuft all indicate that the organisms entered the capillaries in the lung, and were disseminated through the circulation to various organs.

The necrosis of the parenchymal cells in the lungs and kidney adjacent to the fungus may indicate the production of a toxin liberated during fungal proliferation in vivo as suggested by Evans and Winner (1954) and Chitale and Bhende (1965). Winner (1960) has suggested that blockage of blood vessels may be a contributory but not the sole cause of death, because large doses of killed $C$. albicans are not lethal for rabbits.

In the lung and kidney lesions it was noted that the mycelial form of the fungus was invading the neighbouring tissue. The yeast forms showed no such activity. Although both forms were phagocytosed by the macrophages and polymorphonuclear leucocytes, the yeast forms were rapidly digested while the mycelial forms appeared to invade the cells without any sign of being destroyed. Young (1958) has made a similar observation on mice infected intraperitoneally 
with C. albicans, and Louria et al. (1960) observed that the mycelial forms in the tissues evaded the host defence-mechanism because they emerged from the polymorphonuclear leucocytes after phagocytosis. These observations confirm those of Chitale and Bhende, and are directly opposed to the view held by many workers (Nickerson and Edwards, 1949; Scherr, 1951; Hill and Gebhardt, 1956) that among dimorphic fungi the yeast form is the one more capable of growth in the animal body.

Because both yeast and mycelial forms were present in abscesses in more or less equal numbers, it was not possible to decide which form was responsible for abscess formation. Probably both forms played a part, and the process of conversion of the yeast forms to mycelia may be necessary. Chitale and Bhende observed that for suppuration to occur either the yeast form must multiply luxuriantly or hyphae must be present. The larger the number of total organisms in an abscess, the greater the content of hyphal forms.

The bronchopneumonia produced by intra-tracheal infection resolved rapidly with no residual damage to the lungs. Even with the higher dose $\left(10^{8}\right.$ yeasts), the lesions did not persist for more than 5 days. This confirms the existence of a highly efficient clearance mechanism that probably depends predominantly on phagocytosis, although humoral mechanisms may be operative to a certain extent, because agglutination and fragmentation of the fungus was also seen. Arrest of mycelial growth and of epithelial invasion with fragmentation and destruction of the fungus was seen in all organs, including the kidney where the fungus was finally excreted.

Young (1958), Damodaran and Chakravarty (1964) and Chakravarty et al. (1966) have shown similar excretion by the kidney in mice infected by the intraperitoneal route. Once the fungus was eliminated, the abscesses in the kidney healed by fibrosis and scarring during a period of $1 \mathrm{wk}$.

The minimal inflammatory reaction in the lungs, the rapid disposal of the fungus, the complete resolution of the lung pathology and the failure to produce chronic lesions are indicative of high resistance to $C$. albicans infection in the rabbit.

Winner has demonstrated that inapparent infection with $C$. albicans is common and that antibodies to the organism are often present in normal people and rabbits. Gresham and Burns (1960) attributed resistance of the lungs to the abundance of pulmonary reticulo-endothelial cells capable of phagocytosing the organisms. Evans and Winner observed that fungal proliferation does not seem to stimulate the reticulo-endothelial system to activity, and in our animals there was a complete absence of lesions in the spleen and lymph-nodes although cultures of spleen were positive. The large monocytes seemed to be the cells solely responsible for destruction of the fungus.

Young showed that large monocytes were the first cells to appear after the intraperitoneal injection of C. albicans into the mouse. They were invariably phagocytic and destroyed the fungus by digestion while the polymorphs appeared after $3 \mathrm{hr}$ and exhibited no such phagocytic activity. He suggested that the delay in leucocyte migration might have been due to the production of an inhibitory substance by C. albicans. 


\section{CANDIDA LESIONS IN RABBITS}

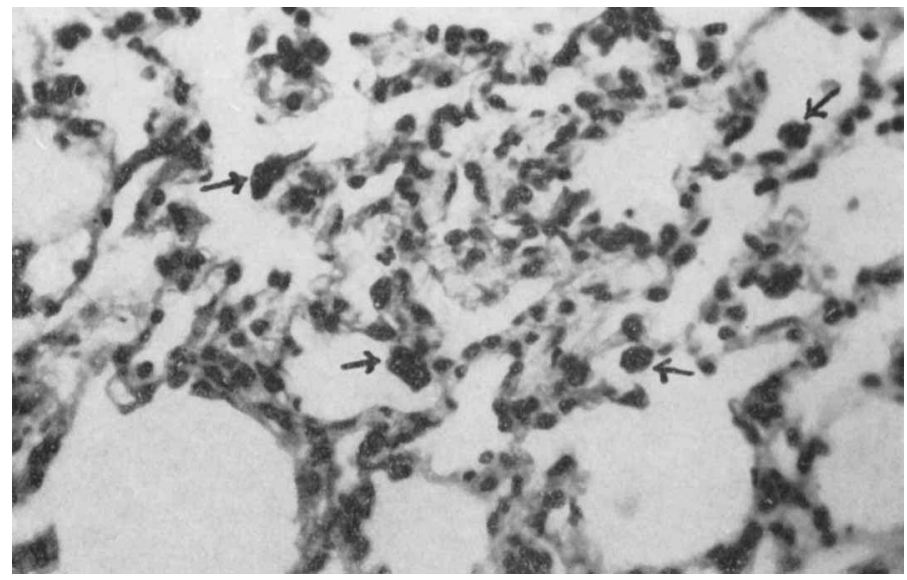

Fig. 1.-Lung, $1 \mathrm{hr}$ after injection of $5 \times 10^{7}$ organisms, showing yeast forms phagocytosed by macrophages (arrows) in the alveoli. Periodic acid-Schiff with haematoxylin (PASH). $\quad \times 270$.

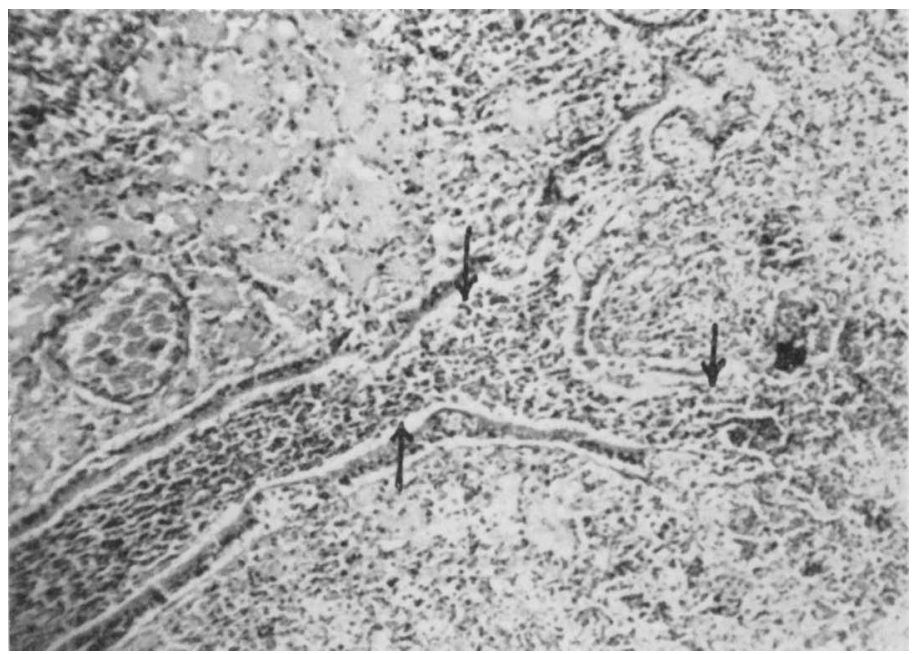

FIG. 2.-Lung, $48 \mathrm{hr}$ after injection of $10^{8}$ organisms, showing bronchopneumonia with exudate filling the bronchus (arrows) and the surrounding alveoli. The bronchus bifurcates and at the right edge shows necrosis of the bronchial wall (arrow). Haematoxylin and eosin. $\times 270$. 


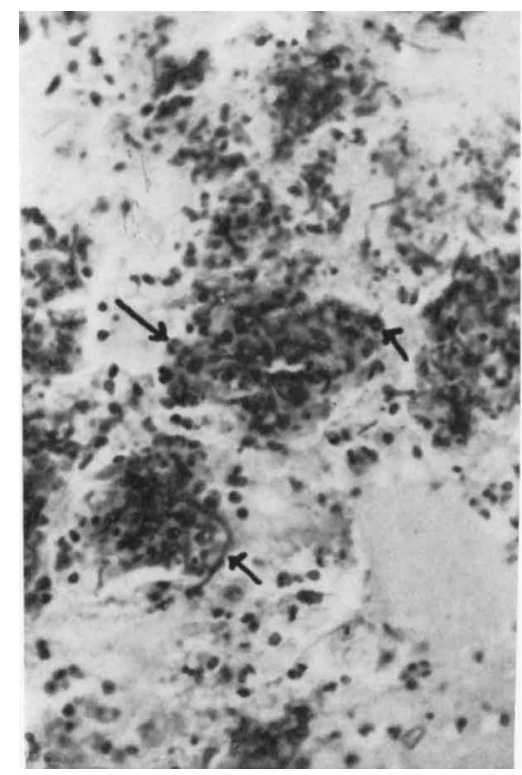

FIG. 3.-Lung of rabbit, 48 hr after injection of $10^{8}$ organisms, showing yeast and mycelial forms (arrows), and deep-stained large round bodies, lying in the alveolar exudate. PASH. $\times 225$.

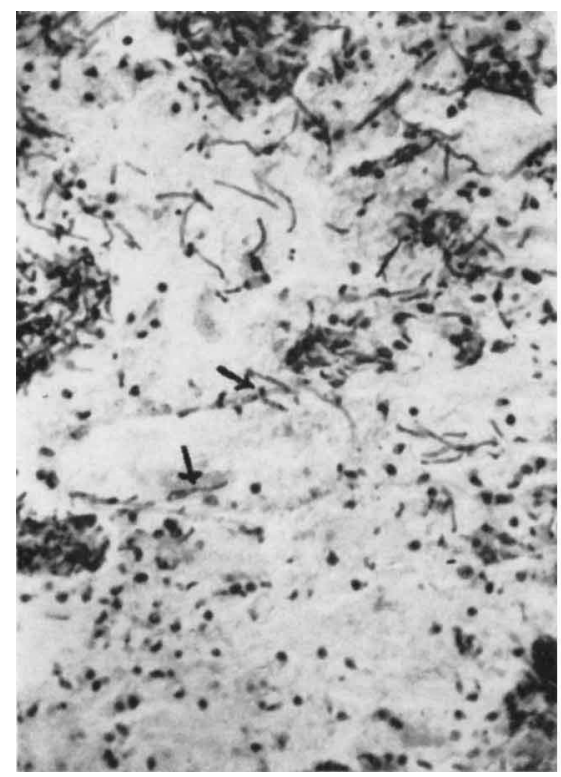

FIG. 5.-Lung (same animal as fig. 2), showing mycelia crossing the thin wall of a blood vessel (arrows). PASH. $\times 225$.

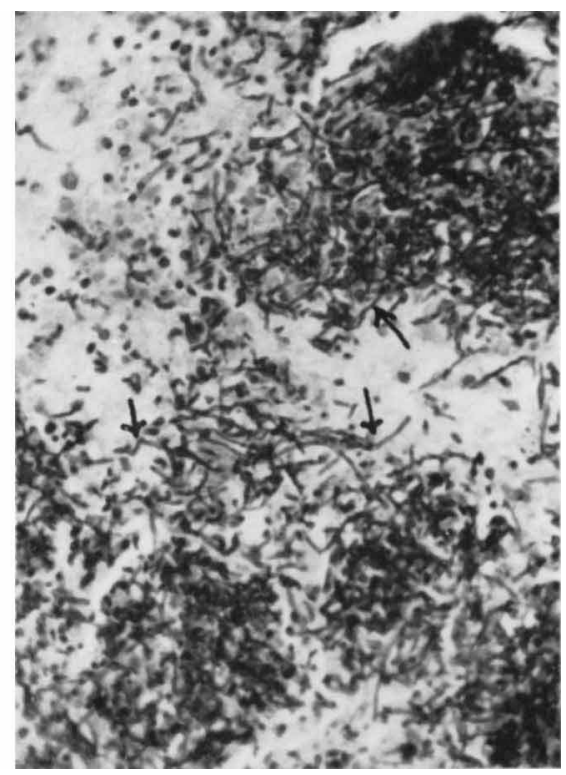

FIG. 4.--Lung (same animal as fig. 2) showing a heavy growth of mycelial forms (arrows) crossing the alveolar septa and small blood vessels. PASH. $\times 225$.

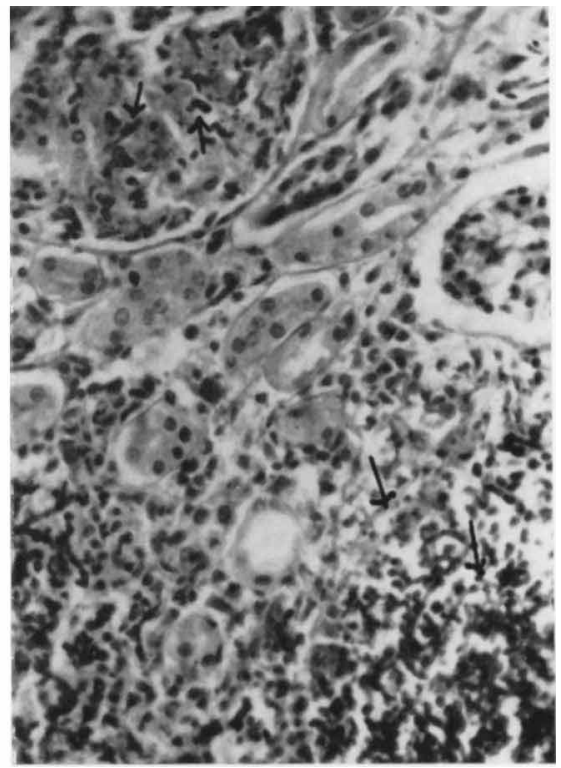

FIG. 6.-Kidney, 3 days after injection of $10^{8}$ organisms, showing emergence of short mycelial forms from the capillaries of the glomerular tuft (arrows) and areas of tubular necrosis (arrows). PASH. $\times 225$. 
The appearance of a few eosinophils associated with necrosis of the bronchial mucosa, congestion and oedema of the lungs and the death of animals after 48 $\mathrm{hr}$ is highly suggestive of a hypersensitivity reaction. Winner suggested that guinea-pigs that are sensitised to $C$. albicans by inapparent infection, and later succumb to a fatal dose with lesions in the lungs and small numbers of yeasts, possibly die from a hypersensitivity reaction of the anaphylactic type. O'Grady et al. (1967) did not observe responses typical of delayed hypersensitivity when mice were re-inoculated with suspensions of Candida and suggested that the difficulty in demonstrating clear differences between primary and secondary lesions may mean that hypersensitivity develops early and plays a part in moulding the inflammatory response of the primary lesion.

From these findings, it is possible to build up a tentative picture of the pathogenesis of the infection. The rabbit lung responded to moderate doses of C. albicans by cellular and probably by humoral mechanisms which destroyed the bulk of the fungus locally. Some fungus entered the blood vessels and was disseminated to all the organs of the body, being finally excreted by the kidney with production, in some cases, of a transient granuloma which healed by fibrosis. With massive infection this defensive mechanism failed, and this resulted in the production of widespread necrosis in most of the organs and death, possibly due to the action of toxins or to blockage of blood vessels.

\section{SUMMARY}

Groups of rabbits were given intratracheal injections of $5 \times 10^{7}$ or $10^{8}$ yeast-forms of Candida albicans. Morphological transformation from yeasts to mycelia was seen in the tissues within $1 \mathrm{hr}$ of injection. The lungs showed an intense cellular reaction with formation of bronchopneumonia, but this quickly and completely resolved and no progressive lesion developed. Rabbit lung seemed to be highly resistant to $C$. albicans infection.

Fungus entered the blood from the lungs and was disseminated to other viscera. Mycelial forms invaded polymorphonuclear leucocytes, large monocytes, blood vessels and renal tubular epithelium. Proliferation of large monocytes was responsible for fragmentation and destruction of the fungus in all the tissues except the spleen, where there was complete absence of reaction although fungal cultures were positive. The fungus was finally excreted by the kidney in which cortical abscesses developed. These healed slowly by scarring with occasional granuloma formation.

\section{REFERENCES}

Ajello, L., George, L. K., Kaplan, W., and Kaufman, L. 1963. Laboratory manual for medical mycology (U.S. Dept. of Health, Education and Welfare), Atlanta, Georgia.

Aoyama, S., AND ATA, S. 1961. Studies on candidiasis in Japan. (Education Ministry of Japan) p. 115.

Chakravarty, S. C., Damodaran, V. N., and Sandhu, R. S. 1966 . Effect of hyaluronidase in experimental candidiasis with special reference to lung lesions. Mycopath. Mycol. appl., 30, 287.

Chitale, A. R., AND Bhende, Y. M. 1965. Experimental moniliasis in rabbits and mice. Indian J. Path. Bact., 8, 89. 
Damodaran, V. N., and Chakravarty, S. C. 1964 . Experimental candidiasis in mice. The effect of hyaluronidase on the production of lesions with particular reference to lungs. Indian J. Chest Dis., 6, 19.

Evans, W. E. D., AND Winner, H. I. 1954. The histogenesis of lesions in experimental moniliasis in rabbits. J. Path. Bact., 67, 531.

Felisati, D., Bastianini, L., and De Mitri, T. 1959. Antibiotics and Candida albicans given by endo-bronchial route. Antibiotics Chemother., 9, 744.

Gresham, G. A., and Burns, M. 1960. Tissue invasion in Candida. In Progress in biological science in relation to dermatology, edited by A. Rook, London, p. 174.

Hill, D. W., AND Gebhardt, L. P. 1956. Morphological transformation of Candida albicans in tissues of mice. Proc. Soc. Exp. Biol. Med., 92, 640.

KUROTChKIN, T. J., AND LIM, C. E. 1933. Experimental broncho-moniliasis in sensitized rabbits. Proc. Soc. Exp. Biol. Med., 31, 332.

Louria, D. B., Fallon, Nancy, AND Browne, H. G. 1960. The influence of cortisone on experimental fungus infections in mice. J. Clin. Invest., 39, 1435.

Nickerson, W. J., AND Edwards, G. A. 1949. Studies on the physiological basis of morphogenesis in fungi. 1. The respiratory metabolism of dimorphic pathogenic fungi. $J$. Gen. Physiol., 33, 41.

O'Grady, F., Pennington, J. H., and Stansfield, A. G., 1967. Delayed hypersensitivity in mouse thigh candidosis. Br. J. Exp. Path., 48, 196.

SCHERR, G. H. 1951. Studies of the dimorphism mechanism in Saccharomyces cereviseae. Mycopath. Mycol. appl., 6, 182.

WinNer, H. I. 1960. Experimental moniliasis in guinea pigs. J. Path. Bact., 79, 420.

Young, G. 1958. The process of invasion and persistence of Candida albicans injected intraperitoneally into mice. J. Infect. Dis., 102, 114. 